\title{
Narrative review of pembrolizumab for the treatment of esophageal cancer: evidence and outlook
}

\author{
Zixian Jin $^{1,2 \#} \wedge$, Jianfei Shen ${ }^{1,2 \#}$, Chunguo Wang ${ }^{1,2 \#}$, Dong Chen ${ }^{1,2}$, Bo Zhang ${ }^{1,2}$, Jian Zhang ${ }^{1,2}$, \\ Jaffer A. Ajani ${ }^{4}$, Jaafar Bennouna ${ }^{5,6}$, Joseph Chao ${ }^{7}$, Harry H. Yoon ${ }^{8}$, Hongyu Zhu ${ }^{1,2}$, Yuhang Ruan ${ }^{1,2}$, \\ Chengchu Zhu ${ }^{1,2}$, Anyi Xü ${ }^{1,3}$
}

${ }^{1}$ Key Laboratory of Minimally Invasive Techniques \& Rapid Rehabilitation of Digestive System Tumor of Zhejiang Province, Linhai, China; ${ }^{2}$ Department of Thoracic Surgery, Taizhou Hospital of Zhejiang Province Affiliated to Wenzhou Medical University, Linhai, China; ${ }^{3}$ Department of Emergency, Taizhou Hospital of Zhejiang Province Affiliated to Wenzhou Medical University, Linhai, China; ${ }^{4}$ Department of GI Medical Oncology, The University of Texas MD Anderson Cancer Center, Houston, Texas, USA; ${ }^{5}$ Department of Medical Oncology, University Hospital of Nantes, France; ${ }^{6}$ Inserm, CRCINA, Université de Nantes, Nantes, France; ${ }^{7}$ Department of Medical Oncology and Therapeutics Research, City of Hope Comprehensive Cancer Center, Duarte, CA, USA; ${ }^{8}$ Division of Medical Oncology, Mayo Clinic, Rochester, MN, USA

Contributions: (I) Conception and design: A Xu, C Zhu; (II) Date analysis and interpretation: Z Jin, J Shen, C Wang, JA Ajani, J Bennouna, HH Yoon; (III) Date check: All authors; (IV) Manuscript writing: Z Jin, J Shen, C Wang, D Chen; (V) Manuscript modification: JA Ajani, J Bennouna, J Chao, HH Yoon, Z Jin, A Xu, C Zhu; (VI) Final approval of manuscript: All authors.

"These authors contributed equally to this work.

Correspondence to: Chengchu Zhu. Key Laboratory of Minimally Invasive Techniques \& Rapid Rehabilitation of Digestive System Tumor of Zhejiang Province, Linhai, China; Department of Thoracic Surgery, Taizhou Hospital of Zhejiang Province affiliated to Wenzhou Medical University, Linhai 317000, China. Email: zhucc@enzemed.com; Anyi Xu. Key Laboratory of Minimally Invasive Techniques \& Rapid Rehabilitation of Digestive System Tumor of Zhejiang Province, Linhai, China; Department of Emergency, Taizhou Hospital of Zhejiang Province affiliated to Wenzhou Medical University, Linhai, China. Email: anyi054@163.com.

Objective: Based on the current evidence, review the efficacy and safety profile of pembrolizumab, along with its shortcomings, in an effort to define future research directions.

Background: The survival outcome of esophageal cancer (EC) is poor, especially in patients with advanced stage. Palliative surgery, chemotherapy, radiotherapy and chemoradiotherapy have limited efficacy in prolonging the survival time. Currently, immunotherapies, including adoptive cell therapy-based, antibodybased, and vaccine-based therapies, are attracting considerable attention. The mechanism of immunotherapy lies in the modification of immune response and prevention of immune escape. Immunomodulatory agents can block the programmed cell death protein 1/programmed death-ligand 1 (PD-1/PD-L1) pathway, thereby allowing lymphocytes to attack tumor cells. This class of drugs has the potential to treat a variety of tumors and may substantially improve overall survival (OS) in some patients. Multiple clinical trials have shown that pembrolizumab has good efficacy and safety, enhances the EC treatment paradigm, and has even become the first-line treatment of choice for patients with PD-L1-positive recurrent or metastatic EC.

Methods: We reviewed the results of clinical trials of pembrolizumab for EC and gastroesophageal cancer presented at Embase, PubMed, the American Society of Clinical Oncology (ASCO) annual meetings, and the Cochrane Central Register of Controlled Trials.

Conclusions: Pembrolizumab has good efficacy and tolerability profiles, and has emerged as a second-line option for the treatment of PD-L1-positive locally advanced or metastatic ESCC. Pembrolizumab has many promising applications, and further investigations into its mechanisms should be conducted.

Keywords: Esophageal cancer (EC); pembrolizumab, programmed death-ligand 1 (PD-1); programmed cell death protein 1 (PD-L1); immune checkpoint inhibitors

$\wedge$ ORCID: 0000-0001-7303-5595. 
Submitted Feb 10, 2021. Accepted for publication Jun 28, 2021.

doi: 10.21037/atm-21-2804

View this article at: https://dx.doi.org/10.21037/atm-21-2804

\section{Introduction}

Esophageal cancer (EC) is the $6^{\text {th }}$ leading global cause of cancer mortality, with approximately 400,000 deaths annually $(1,2)$. Currently, specific treatment regimens for EC are selected based on the stage and location of the tumor as well as the patient's physiologic condition and social support available to the patient (3). Most early-stage ECs can be treated by surgery or endoscopy. However, due to earlystage EC often lacking specific symptoms and the tendency of many people to neglect routine health check-ups, in most cases, the EC has already advanced by the time it is diagnosed. For resectable EC, surgery alone does not achieve satisfactory results, and surgery combined with radiotherapy and/or chemotherapy is currently the mainstay of treatment $(3,4)$. However, the efficacy of the treatment depends highly on the molecular biology of EC, the patient's general condition, and the sensitivity to conventional radiotherapy/ chemotherapy/surgery is often suboptimal (3). A study analysis based on the SEER database showed that the 5 -year survival rate after resectable esophageal cancer was only $40.5 \%$ (5). Therefore, there is an urgent need to identify and develop new treatment strategies to improve the clinical outcomes of patients with EC.

Pembrolizumab is active not only in certain tumor types but also for tumors with specific genetic abnormalities (6). Tumor cells that have many mutations due to a deficiency in DNA mismatch repair (dMMR), which often results in microsatellite instability high (MSIH), produce multiple mutant proteins. Programmed cell death protein 1 (PD-1) inhibitors can facilitate the elimination of such tumors by the immune system by inhibiting immune checkpoint $(7,8)$. Several early trials suggest that immune checkpoint inhibitor like pembrolizumab may have some efficacy for the treatment of advanced EC and the neoadjuvant treatment of EC (9-14). Pembrolizumab was approved by the United States Food and Drug Administration (USFDA) in 2014 for the second-line or multi-line treatment of patients with any unresectable or metastatic solid tumor; in 2019, it was approved for the second-line treatment of patients with programmed death-ligand 1 (PD-L1)-positive, locally advanced or metastatic esophageal squamous cell carcinoma (ESCC) (15). Since 2020, the results of the PALACE-1 trial for resectable EC have been announced, and phased data from the NCT02844075 trial and KEYNOTE-590 trial have also been published $(9,10,14)$. In March 2021, the USFAD approved pembrolizumab as a first-line treatment for advanced esophageal cancer.

Therefore, at the time of these major events, it is important to review the Phase I-III data of pembrolizumab in EC, review the efficacy and safety profile of pembrolizumab, and point out its shortcomings in order to determine the direction of future research.

We present the following article in accordance with the Narrative Review reporting checklist (available at https:// dx.doi.org/10.21037/atm-21-2804).

\section{Methods}

We reviewed the results of clinical trials of pembrolizumab for esophageal cancer and gastroesophageal cancer presented at Embase, PubMed, the American Society of Clinical Oncology (ASCO) annual meetings, and the Cochrane Central Register of Controlled Trials. The latest articles or conference abstracts of representatives were selected and included in this analysis. The literature included in the analysis was not limited to publication in English, and there was no time limit. Non-prospective clinical studies were not included.

\section{Preclinical evidence}

PD-1 is a negative regulatory molecule expressed by activated lymphocytes $(16,17)$. By binding to its ligand (PD-L1 and PD-L2), PD-1 can down-regulate lymphocyte activation and inhibit anti-tumor immune response; however, this also prevents the killing of cancer cells (17-19). Malignant tumors, including esophageal cancer, can express PD-L1 binding to PD-1, which initiates programmed death of $T$ lymphocytes, thereby reducing the effector activity of $\mathrm{T}$ lymphocytes and terminating the immune response (20-22).

Pembrolizumab is a highly selective, humanized monoclonal immunoglobin $(\mathrm{Ig}) \mathrm{G} 4-\kappa$ antibody that blocks the interaction between PD-1 and its ligand PDL1 while preserving the antitumor function of the host T cells. Unlike other subclasses of IgG, IgG4 has a low 
Table 1 Clinical trials on preoperative neoadjuvant therapy for resectable esophageal cancer in which pembrolizumab was involved

\begin{tabular}{lcclccc}
\hline Study & Phase & N & Treatment & pCR (\%) & OS (\%) & AEs $(\geq 3, \%)$ \\
\hline PALACE-1 & I & 20 & Pembrolizumab + chemoradiotherapy & 55.6 & 65 & NR \\
NCT02844075 & II & 28 & Pembrolizumab + chemoradiotherapy & 46.1 & 6 mo: 89.3; 12 mo: 80.8; 18 mo: 73.1 & NR \\
\hline
\end{tabular}

$\mathrm{N}$, number; pCR, pathological response rate; AEs, adverse reactions; OS, overall survival; NR, not reported; \%, percent; mo, month.

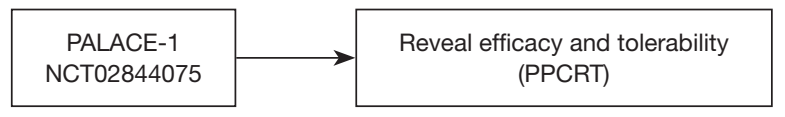

Figure 1 Historical contribution of pembrolizumab to preoperative treatment of resectable esophageal cancer. PPCRT, preoperative pembrolizumab combined with chemoradiotherapy.

affinity for Fc receptors and C1q; therefore, the binding of pembrolizumab to $\mathrm{PD}-1$ on $\mathrm{T}$ cells does not activate the complement system (17). Pembrolizumab can blocks the PD-1/PD-L1 pathway, reactivates tumor-specific cytotoxic T lymphocytes in the tumor microenvironment, and restore anti-tumor immune activity $(19,23)$. As pembrolizumab is cleared from the circulation via a nonspecific metabolic pathway, pharmacokinase-related drug interactions do not occur; the clearance rate is approximately $0.2 \mathrm{~L} / \mathrm{day}$, and the half-life is about 25 days.

\section{Treatment of resectable EC}

At present, the mainstay of treatment for resectable EC is neoadjuvant therapy in combination with surgical resection. However, due to lack of high-quality evidence, the standard adjuvant treatment strategies for EC remain controversial. Therefore, the role of pembrolizumab in the neoadjuvant treatment of EC has been investigated (Table 1, Figure 1).

A phase I trial (PALACE-1) conducted by Li et al. explored the safety of preoperative pembrolizumab combined with chemoradiotherapy for ESCC (9). Twenty patients with resectable ESCC received preoperative pembrolizumab with concurrent chemoradiotherapy (PPCT), regardless of PD-1 status. Preoperative therapy included carboplatin, paclitaxel, radiotherapy, and pembrolizumab. Within 4-6 weeks following preoperative therapy, the patients underwent surgery. The primary endpoint was safety. Common adverse reactions during neoadjuvant treatment included lymphopenia (100\%), anemia (80\%), esophagitis (55\%), and pneumonia (20\%). Adverse events (AEs) of grade 3 or higher (mainly lymphopenia) were observed in 13 patients $(13 / 20,65 \%)$, and 1 patient died of esophageal hemorrhage. PPCT was found to be safe and did not delay surgery. Eighteen patients underwent surgery within 4-9 weeks, and 1 patient did not undergo surgery due to disease progression. A pathological complete response (pCR) rate of $55.6 \%$, an $\mathrm{R} 0$ resection rate of $94 \%$, and a major pathologic response ( $\mathrm{mPR}$ ) rate of $89 \%$ were attained. Postoperatively, the treatment protocol continued to work, and no recurrence was observed in any of the patients who received $\mathrm{R} 0$ resection after a median follow-up of 6.6 months. The researchers also found that PD-L1 expression was not associated with pathological remission, while TCF-1+CD8+ T-cell infiltration was significantly higher in tumors with pCR than in those without pCR.

In Hong et al.'s phase II trial (NCT02844075), 28 patients with resectable ESCC were enrolled (10). Patients received concurrent neoadjuvant chemotherapy (weekly paclitaxel and carboplatin), radiotherapy, and pembrolizumab followed by surgery. Two patients did not undergo surgery due to death (hematemesis) and withdrawal of consent. Twelve of 26 patients (the other two patients did not undergo surgery due to death (hematemesis) and withdrawal of consent.) achieved pCR at the primary site (pCR rate: $46.1 \%$; $95 \%$ CI: $28.0-64.6 \%)$. The 6-, 12-, and 18-month overall survival (OS) rates were $89.3 \%, 80.8 \%$, and $73.1 \%$, respectively. Neutropenia (50\%) and elevated liver transaminases (30.8\%) were the most commonly occurring toxicities, and 2 patients died from severe lung injury following surgery.

The above studies offer early new evidence to support further investigation of neoadjuvant immunotherapy in the treatment of EC (Figure 1). However, by comparing the pCR rate of CROSS trial and NEOCRTEC5010 trial after neoadjuvant chemoradiation (49\% and 43.5, respectively) $(24,25)$, we found that the pCR rate of neoadjuvant immunotherapy (pembrolizumab) combined with chemoradiation did not show an advantage over neoadjuvant chemoradiation. However, the study of preoperative checkpoint inhibition in EC is at early stage. Furthermore, the toxicities (especially immune-associated 
Table 2 Clinical trials on advanced esophageal cancer in which pembrolizumab was involved

\begin{tabular}{|c|c|c|c|c|c|c|c|c|}
\hline Study & Phase & $\mathrm{N}$ & Treatment & OS (m, mo) & PFS (m, mo) & ORR (\%) & $\mathrm{DOR}(\mathrm{m}, \mathrm{mo})$ & AEs $(\geq 3, \%)$ \\
\hline KEYNOTE-180 & II & 121 & Pembrolizumab & 5.8 & 2 & 9.9 & $\mathrm{Nr}$ & 12.4 \\
\hline KEYNOTE-181 & III & 628 & $\begin{array}{c}\mathrm{T}_{1}: \text { chemotherapy; } \mathrm{T}_{2}: \\
\text { pembrolizumab }\end{array}$ & $\mathrm{T}_{1}: 7.1 ; \mathrm{T}_{2}: 7.1$ & $\mathrm{~T}_{1}: 2.1 ; \mathrm{T}_{2}: 3.4$ & $\begin{array}{l}\mathrm{T}_{1}: 6.7 \\
\mathrm{~T}_{2}: 13.1\end{array}$ & NR & $\begin{array}{c}\mathrm{T}_{1}: 40.9 \\
\mathrm{~T}_{2}: 18\end{array}$ \\
\hline
\end{tabular}

$\mathrm{N}$, number; OS, overall survival; PFS, progression-free survival; ORR, overall response rate; DOR, duration of response; m, median; mo, month; \%, percent; Nr, not reached; NR, not reported; AEs, adverse reactions.

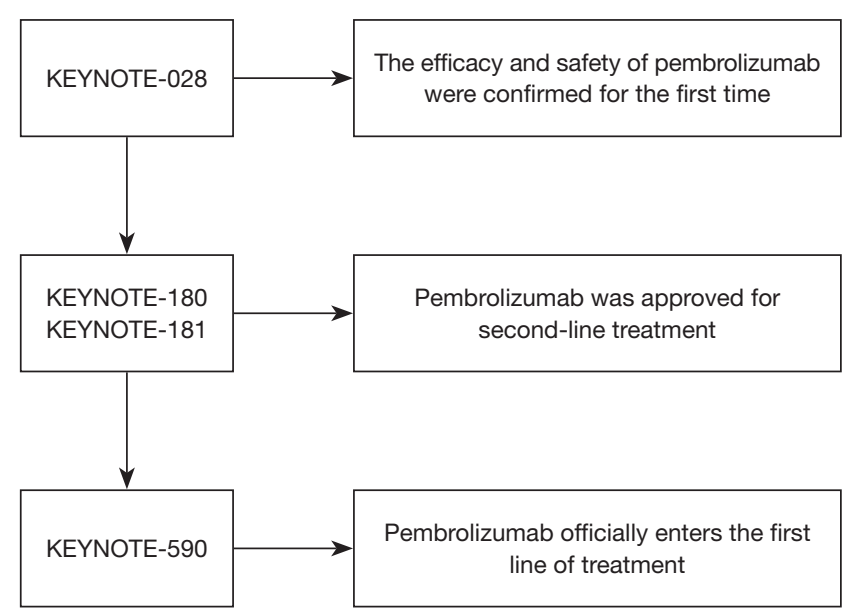

Figure 2 The historical progression of pembrolizumab into the first- and second-line treatment of advanced/recurrent/metastatic non resectable esophageal cancer.

pneumonia) of neoadjuvant concurrent chemoradiotherapy plus immunotherapy, are to be considered moving forward. Therefore, many controversies still surround the use of neoadjuvant immunotherapy for EC.

\section{Treatment of advanced EC}

During the early stage, EC lacks typical symptoms; thus, in most cases, the disease has already progressed to an advanced stage at diagnosis. In some other cases, the cancer progresses to an advanced stage after the patient has received treatment. Consequently, the long-term survival rate of patients with EC is low. Previous treatment of patients with advanced EC has mostly focused on radiotherapy, chemotherapy, chemoradiotherapy, and/ or palliative resection, which significantly lower patients' quality of life while achieving temporary disease control $(3,5)$. Immune checkpoint inhibitors have been incorporated into the treatment of malignancies including EC, gastric cancer, lung cancer, and colorectal cancer (26-29). There is growing evidence that immune checkpoint inhibitors such as pembrolizumab could play important roles in the treatment of patients with advanced EC (Table 2, Figure 2).

\section{Phase I clinical trials}

In KEYNOTE-028 a phase IB study of patients with PDL1-positive advanced solid tumors, 28 eligible patients with ESCC or adenocarcinoma of the esophagus or gastroesophageal junction for whom standard therapy had failed were given pembrolizumab $10 \mathrm{mg} / \mathrm{kg}$ every 2 weeks (11). Twenty-three PD-L1-positive [combined positive score (CPS) $\geq 1 \%$ ] patients were entered into the final analysis. The median follow-up was 7 months (range, 1 to 33 months). The overall response rate (ORR) was $30 \%$ (95\% CI: $13-53 \%$ ); the median duration of response was 15 months (range, 6-26 months); the median PFS was 1.8 months (95\% CI: 1.7-2.9 months); the 12-month progression-free survival (PFS) rate was $22 \%$; the median OS was 7.0 months (95\% CI: 4.3-17.7 months); and the 12 -month OS rate was $40 \%$. Grade 3 toxicities were reported in $39 \%$ of patients, but no grade 4 events were recorded. The authors concluded that pembrolizumab demonstrated similar efficacy and safety profile in PDL1-positive advanced EC as it did in other tumors. KEYNOTE-028 was the first study to demonstrate the activity and safety of pabolizumab in the treatment of advanced EC (Figure 2). 


\section{Phase II clinical trials: multiline therapies}

The phase II, open-label, non-randomized study KEYNOTE-180 enrolled 121 patients with advanced, metastatic EC that had progressed after 2 or more lines of therapy (12). Among the patients, 63 (52.1\%) had ESCC and $58(47.9 \%)$ had adenocarcinoma. Pembrolizumab, $200 \mathrm{mg}$, was administered intravenously every 3 weeks. After treatment, the tumor burden decreased in 43 of 106 patients. The median PFS was 2.0 months $(95 \%$ CI: 1.9-2.1 months), and the 6- and 9-month PFS rates were $16 \%$ (95\% CI: $10-23 \%$ ) and 9\% (95\% CI: 5-16\%), respectively. The median OS was 5.8 months (95\% CI: 4.5-7.2 months), and the 6- and 12-month OS rates were 49\% (95\% CI: $40-57 \%$ ) and $28 \%$ (95\% CI: $20-37 \%$ ), respectively. The ORR was $14.3 \%$ (95\% CI: $6.7-25.4 \%)$ in patients with ESCC (52.1\%), 13.8\% (95\% CI: 6.1-25.4\%) in PD-L1-positive patients, and 6.3\% (95\% CI: 1.8-15.5\%) in PD-L1-negative patients. PFS was similar across all subgroups, while OS was longer for the ESCC and PDL1-positive patients than for the PD-L1-negative patients. In this phase II clinical trial, pembrolizumab demonstrated antitumor activity of clinical significance regardless of tumor histology.

\section{Phase III clinical trials: second-line therapies}

The multi-center, randomized, open-label, phase III study KEYNOTE-181 enrolled 628 patients with recurrent locally advanced or metastatic EC (or with Siewert type I esophagogastric junction (EGJ) adenocarcinoma) whose cancer progressed on or after 1 prior line of systemic treatment (13). The patients were randomized $(1: 1)$ to receive either pembrolizumab $200 \mathrm{mg}$ intravenously (IV) every 3 weeks or the investigator's choice from the following regimens: paclitaxel, docetaxel, or irinotecan. Randomization was stratified by geographic region and histologic subtype. The primary efficacy outcome measure was OS of patients with ESCC, patients with tumors expressing a high level of PD-L1 (CPS $\geq 10)$, and all intention-to-treat patients. The secondary endpoints were PFS, ORR, and treatment safety/tolerance.

In the pembrolizumab and chemotherapy groups, the median follow-up period was 7.1 and 6.9 months, respectively. OS was longer with pembrolizumab than with chemotherapy ( $\mathrm{n}=222)$ for patients with CPS $\geq 10$ [median: 9.3 vs. 6.7 months; hazard ratio (HR), 0.69; 95\% CI: 0.52-0.93; $\mathrm{P}=0.0074]$. The estimated 12 -month OS rate was $43 \%$ with pembrolizumab versus $20 \%$ with chemotherapy. The incidence of overall treatment-related AEs (64\% vs. 86\%) was significantly lower in the pembrolizumab group than in the chemotherapy group, as was that of grade 3-5 treatmentrelated AEs (18.2\% vs. 40.9\%). Subgroup analysis showed that Asian populations and patients with ESCC could benefit more from immunotherapy, and there were also notable differences in the efficacy of immunotherapy in terms of geographic region and pathological type, which should be carefully considered in future clinical studies on EC.

The authors concluded that pembrolizumab prolonged OS compared with chemotherapy as a second-line therapy for advanced EC in patients with PD-L1 CPS $\geq 10$, with superior safety. Accordingly, the USFDA has approved pembrolizumab as a standard second-line treatment for ESCC with positive PD-L1 expression (CPS $\geq 10)$ in 2019 (Figure 2).

\section{Phase III clinical trials: first-line therapies}

KEYNOTE-590 was a randomized, double-blind phase III study of pembrolizumab plus chemotherapy versus chemotherapy alone in patients with locally advanced/ unresectable or metastatic adenocarcinoma, ESCC, or Siewert type 1 gastroesophageal junction (EGJ) adenocarcinoma (14). The chemotherapy regimen was 5 -fluorouracil combined with cisplatin (FP). At the data cutoff, 749 patients (including 548 patients with ESCC) were randomized (373 pembrolizumab plus chemotherapy; 376 placebo plus chemotherapy). Pembrolizumab plus chemotherapy was superior to placebo plus chemotherapy with respect to OS for patients with ESCC (HR 0.72) or CPS $\geq 10$ (HR 0.62), and especially so for patients with ESCC and CPS $\geq 10$ (median: 13.9 vs. 8.8 months; HR $0.57 ; \mathrm{P}<0.0001)$. In the pembrolizumab plus chemotherapy group $(n=373)$ and the placebo plus chemotherapy group $(\mathrm{n}=376)$, the confirmed ORR was $45.0 \%$ vs. $29.3 \%$ $(\mathrm{P}<0.0001)$, respectively, with a median duration of response of 8.3 vs. 6.0 months $(\mathrm{P}<0.0001)$, respectively. For the total study population, the OS of the pembrolizumab plus chemotherapy group was significantly longer than that of the placebo plus chemotherapy group (12.4 vs. 9.8 months, $\mathrm{P}<0.0001$, HR 0.73). Thus, when used in a first-line setting, pembrolizumab combined with chemotherapy (5-fluorouracil and cisplatin) provided good efficacy and safety for patients with advanced EC and EGJ adenocarcinoma.

The results of this trial pave the way for pembrolizumab 
combined with chemotherapy to be the first-line treatment for advanced ESSC even on March 22, 2021, the USFDA generically approved pembrolizumab in combination with platinum and fluorouracil chemotherapy for first-line use in advanced EC and EGJ cancers, regardless of histological or tumor PD-L1 status (Figure 2). However, data from the KEYNOTE-590 trial also showed that in patients with PD-L1 CPS $<10$ ( $n=347)$, the combination therapy did not provide a statistically significant survival benefit compared with chemotherapy alone [HR 0.86; 95\% CI: 0.68-1.10; mOS 10.5 (9.7-13.5) vs. $10.6(8.8-12.0)]$. The data suggest a lack of benefit from the addition of pembrolizumab to platin/FP in CPS $<10$, despite the FDA approval.

\section{Pembrolizumab-related AEs}

The use of pembrolizumab prompts the immune system to attack normal cells, which may result in autoimmune diseases. Common side effects of the drug include pruritus, rash, cough, fever, nausea, and constipation. Moreover, AEs to pembrolizumab, such as immune-associated pneumonia and acute respiratory distress syndrome, should not be underestimated $(15,30)$.

In the PALACE-1 study, the incidence of AEs of grade 3 or higher was $65 \%$, with the most common $\mathrm{AE}$ being lymphopenia (12/13, 92\%) (9). One patient died of esophageal rupture. In the KEYNOTE-028 trial, 9 patients (39.1\%) suffered AEs, the most common of which were rash, loss of appetite, and decreased lymphocyte count (11). Although 4 patients experienced grade 3 AEs, no AEs of grade 4 or higher or deaths occurred.

Among the 121 patients in the KEYNOTE-180 trial, 70 patients experienced different degrees of AEs, with the most common AEs being fatigue, rash, pruritus, hypothyroidism, and diarrhea (12). Only 5 patients discontinued treatment because of AEs. Fifteen patients suffered from AEs of grade 3 to 5 , and 1 died of treatment-related pneumonia. Twenty-five patients experienced immune-related adverse events (irAEs). The incidence of drug-related AEs of all-grades was 64\% with pembrolizumab compared to $86 \%$ with chemotherapy, and grade 3 to 5 drug-related AEs occurred at a rate of $18 \%$ and $41 \%$, respectively. The most commonly occurring AEs in the pembrolizumab group were fatigue, hypothyroidism, decreased appetite, asthenia, and nausea, while fatigue, diarrhea, and hematologic toxicity were most common in the chemotherapy group. Treatment-related AEs led to the discontinuation of therapy in approximately $6 \%$ of patients in each group and to the deaths of 5 patients in each group. Immune-mediated AEs and infusion reactions occurred in $23.2 \%$ of patients in the pembrolizumab group and in $7.4 \%$ of patients in the chemotherapy group. In the ongoing KEYNOTE-590 trial, the incidence of AEs of grade 3 or higher was higher in the pembrolizumab plus chemotherapy group (72\%) than in the chemotherapy group (68\%), as was the rate of treatment discontinuation (19\% vs. 12\%) (14).

Therefore, whether pembrolizumab is used alone or in combination with chemotherapy, compared with chemotherapy alone, there is no advantage in tolerability. Furthermore, the toxicities of neoadjuvant concurrent chemoradiotherapy plus immunotherapy, especially the occurrence of immune-associated pneumonia, are rather concerning.

\section{Discussion}

Pembrolizumab has demonstrated survival benefit and good tolerability in the treatment of various cancers, and the USFDA has approved it as an immune checkpoint inhibitor for any unresectable or metastatic solid tumor with a specific genetic signature. As shown in many clinical trials, pembrolizumab is effective and has good tolerability for the treatment of resectable or advanced EC.

However, in KEYNOTE-180, the ORR of patients with PD-L1-positive (CPS $\geq 10$ ) advanced EC was significantly higher than that of patients with PD-L1-negative (CPS $<10)$ EC (12). The phase III KEYNOTE-181 found that among patients with $\mathrm{PD}-\mathrm{L} 1$-positive advanced EC, the OS rate was significantly higher in the pembrolizumab group than in the chemotherapy group; however, a significant advantage of pembrolizumab treatment was not found among patients with PD-L1-negative EC (13). The subgroup analysis also showed that the OS rate was significantly higher in the pembrolizumab group than in the chemotherapy group among PD-L1-positive (particularly, with CPS $=10$ or higher) patients with ESCC, whereas there was no significant difference in OS between the pembrolizumab and chemotherapy groups among PD-L1positive patients with esophageal adenocarcinoma (13). Thus, pembrolizumab may only have unique benefits for specific patients (for instance, patients with PD-L1positive with CPS $=10$ or higher ECSS). However, both the KEYNOTE-180 and KEYNOTE-181 trials defined PD$\mathrm{L} 1$ positivity as a CPS score of $\geq 10$ (i.e., $\mathrm{PD}-\mathrm{L} 1$ detected in at least $10 \%$ of tumor or inflammatory cells or in the lung 
mesenchyme), and the optimal cut-off for CPS deserves further exploration and validation. Also, some patients with a CPS score of $<10$ also achieved a clinical response after pembrolizumab treatment; thus, PD-L1 expression may be only 1 of the indications for pembrolizumab treatment, rendering PD-L1 testing alone to be insufficient for patient selection (31). Based on the currently available clinical evidence, the USFDA has approved pembrolizumab as a second-line treatment for patients with PD-L1-positive advanced, metastatic, or progressive ESCC, making it the first anti-PD-1 therapy to be approved for the treatment of ESCC. Other biomarkers, such as high MSI or mismatch repair deficiency, are also under constant investigation (32).

Interesting early results have become available from trials of the combination of pembrolizumab with chemotherapy. The phase III trial KEYNOTE-590 found that pembrolizumab plus chemotherapy demonstrated a survival advantage over chemotherapy combined with placebo in patients with advanced EC in the overall population of any PD-L1 status, but this benefit appeared to be derived primarily from patients with a high expression of PD-L1 (CPS $\geq 10$ ) (14). In particular, among patients with ESCC and CPS $\geq 10$, the combination treatment showed an even more obvious advantage over advantage over chemotherapy plus placebo. The objective response rate was also greater in the combination group than in the chemotherapy plus placebo. This result led the FDA to approve pembrolizumab in combination with chemotherapy for the first-line treatment of locally advanced unresectable or metastatic esophageal and gastroesophageal junction cancers. Data published from the KEYNOTE-181 and KEYNOTE-590 trials showed that patients with esophageal cancer with either pembrolizumab combined immunotherapy or pembrolizumab monotherapy had longer long-term survival compared with chemotherapy alone when CPS was not differentiated. This survival advantage was more significant in patients with ESCC and CPS $\geq 10$. For patients with advanced esophageal cancer with recurrence or metastasis after first-line or multi-line treatment, conventional radiotherapy, chemotherapy or radiotherapy are difficult to exert efficacy. Pembrolizumab gives these patients new light.

As with advanced EC, we have also witnessed the shortterm treatment efficacy and clinical tolerability of treatment combinations with pembrolizumab in resectable EC from the PALACE- 1 trial and the NCT02844075 trial $(9,10)$. For patients with resectable esophageal cancer, there are new options for preoperative treatment. However, comparing the two studies with the Cross trial and
NEOCRTEC5010 trial $(24,25)$, we found that the pCR rates of the combination of neoadjuvant pembrolizumab and chemotherapy did not significantly improve over neoadjuvant chemotherapy, even patients had to bear the additional side effects of the immune checkpoint inhibitors. The PALACE-1 study showed that PD-L1 expression was not associated with pathological response, while TCF$1+$ and CD8+ T cell infiltrates in tumors with PCR were significantly higher than those in tumors without PCR (9). Therefore, the method of predicting whether or not resectile EC patients will achieve PCR after neoadjuvant pembrolizumab combined with chemoradiotherapy remains unclear and needs further investigation.

Additionally, an ongoing phase Ib-2 trial (CPMGAH22-05) also revealed that margatuximab in combination with pembrolizumab had good tolerability and efficacy in the treatment of human epidermal growth factor receptor 2 (HER2) positive gastroesophageal adenocarcinoma (33). KEYNOTE-975, a randomized, multicenter, double-blind, phase III study investigating the possibility of using pembrolizumab in the definitive chemoradiation setting, is currently in the patient recruitment stage (34). Patients with inoperable locally advanced, but non-metastatic ESCC or adenocarcinoma or Siewert type 1 adenocarcinoma of the EGJ will be randomly assigned $(1: 1)$ to receive pembrolizumab plus definitive chemoradiotherapy (dCRT) or a placebo plus dCRT. The trial will attempt to determine whether pembrolizumab combined with dCRT can prolong OS and improve tolerability in patients with advanced EC compared to dCRT alone. The trial has not distinguished between different PD-L1 expression status during patient enrolment, and again, PD-L1 positivity has been defined as a CPS of $\geq 10$ (34). Other ongoing clinical trials including NCT02844075, NCT02998268, NCT03322267, NCT04389177, and NCT04435197 are expected to yield further clinical evidence to illustrate the efficacy and tolerability of pembrolizumab.

Other immune checkpoint inhibitors (e.g., nivolumab, tislizumab, toripalimab, sintilimab and camrelizumab as well combination of nivolumab and ipilimumab) targeting PD-1 (and CTLA4) have also shown notable antitumor potential. The follow-up findings of a phase III clinical trial revealed that nivolumab demonstrated significantly better overall efficacy than chemotherapy in patients with advanced ESCC that was refractory or who were intolerant to previous chemotherapy, regardless of PD-L1 expression (35). The CheckMate-577 trial also demonstrated that nivolumab prolonged the 
PFS of patients with resected EC following neoadjuvant chemoradiotherapy (36). Camrelizumab and tislelizumab have also shown promising results in their respective clinical trials (ESCORT and Rationale 302trial) (37).

Despite the remarkable advances shown by pembrolizumab in terms of efficacy and tolerability, its shortcomings should not be ignored. First, as an immune checkpoint inhibitor, pembrolizumab is a systemic treatment drug that not only acts in tissues affected by the tumor but in all tissues in the human body. Therefore, a thorough evaluation of the patient is required before pembrolizumab is used. Severe infusion-related reactions were reported in patients receiving pembrolizumab (11-13). For severe infusion reactions, the infusion must be discontinued and pembrolizumab discontinued. Patients with mild or moderate infusion reactions can continue to receive pembrolizumab under close monitoring; at the same time, antipyretic and analgesic anti-inflammatory drugs and antihistamines can be considered for prevention. Also, irAEs in patients treated with pembrolizumab cannot be ignored, such as dermatologic toxicity, endocrinopathies, colitis, pneumonitis and liver toxicity $(11-14,19,38)$. In the KEYNOTE-180 trial, 70 of 121 patients experienced AEs of varying severity, including irAEs in 25 cases (12). In the KEYNOTE-181 trial, the pembrolizumab arm had a lower overall rate of AEs than the chemotherapy arm; the pembrolizumab arm did, however, have a higher proportion of immune-mediated and infusion reactions (23.2\% vs. 7.4\%) (13). Similarly, in the KEYNOTE-590 trial, the incidence of AEs of grade 3 or higher was higher in the pembrolizumab plus chemotherapy group (72\%) than in the chemotherapy-alone group (68\%) (14). Thus, the immunotherapy showed no advantage in terms of tolerability compared to chemotherapy. A meta-analysis showed that the relative risk of fatal adverse events (FAEs) was independent of tumor type $(\mathrm{P}=0.99)$ (39). The total incidence of FAEs in patients treated with pembrolizumab was $1.2 \%$ (95\% CI: $0.5-2.8 \%$ ) (39). Grade 1-2 irAEs are currently commonly controlled with steroids and other immunomodulators; Grade 3 and 4 irAEs require high doses of glucocorticoids or permanent discontinuation $(40,41)$.

Current evidence does suggest that pembrolizumab is superior in long-term survival compared to chemoradiotherapy. However, it is undeniable that it does not have advantages in terms of AEs, especially in immune-related adverse events. The risk of FAEs with pembrolizumab monotherapy may be similar to that with conventional chemotherapy $(\mathrm{P}=0.35)$ (39). However, when pembrolizumab was used in combination with chemotherapy, the risk of treatment-related mortality increased by 58 percent (39). Moreover, pembrolizumab is expensive and is a significant burden for most patients. Take a $100 \mathrm{mg} / 4 \mathrm{~mL}$ box of Pembrolizumab, which costs $\$ 4,800$ in the US. In Hong Kong, China, the price is HK $\$ 30,000$. In mainland China, it costs $54 \%$ of what it costs in the United States. Therefore, we need to develop and validate a "suite" of feasible biomarkers and establish large-scale databases in large centers for the creation of prognostic prediction models for the treatment of EC with pembrolizumab and for the screening of patients with optimal efficacy and safety profiles $(42,43)$. It has been found that chemotherapy decreases PD-L1 expression, while chemoradiotherapy increases PD-L1 expression in tumor cells $(44,45)$. Therefore, in efforts to build predictive models and develop biomarkers, attention needs to be paid to pembrolizumab as a monotherapy and as a combination treatment with radiotherapy and/or chemotherapy. The role of multidisciplinary collaborative care should also be emphasized further (46).

\section{Questions to be further considered}

What roles do postoperative adjuvant therapy and adjuvant immunotherapy play in the treatment of esophageal cancer? Can you describe the real-world applications of these therapies in your center?

\section{Expert opinion: Ken Kato}

Checkmate-577 shows the benefit of adjuvant use of nivolumab for esophageal cancer. To adapt this for the "Japanese" clinical practice of esophageal cancer, we should take attention to two questions. One is whether nivolumab plays a positive role even after neoadjuvant chemotherapy? Neo-chemo is the standard of care for Japanese Eso Ca patients. The use of radiation before surgery may be the cause of the positive result of nivolumab. There is no evidence of adjuvant Nivo using after neo-chemo-surgery. Another is the difference in the surgical procedures. Radical lymph node dissection has performed in Japan compared to Western countries, and basal survival result was different from Western and Eastern. It is still a question whether Nivo could make difference after surgery with radical LN dissection.

\section{Expert opinion: Harry H. Yoon}

For patients with esophageal squamous cell carcinoma or 
adenocarcinoma who completed trimodality therapy and had residual tumor in the resected specimen, adjuvant nivolumab for one year is the new standard of care. We are now generally providing this treatment in our patients in this setting. However, exploratory analyses of CM-577 in the subgroup with PD-L1 CPS $<5$ [HR 0.89 (0.65-1.22); $\mathrm{n}=295$ ] raises questions and warrants further investigation in future studies.

\section{Expert opinion: Jaafar Bennouna}

The recent publication of the CheckMate 577 raised many questions. The CheckMate 577 is an international randomized multicentric phase III trial comparing in resected stage II, III esophageal and gastro-oesophageal junction cancer nivolumab versus placebo for 1 year. The primary endpoint was Disease Free Survival. Patients have to be initially treated according to the CROSS regimen with neo-adjuvant chemoradiotherapy. Patients were randomized in a 2:1 ratio within 4 to 16 weeks followed surgery. Overall Patients receiving nivolumab had a longer DFS of 22.4 versus 11.0 months in the placebo arm (HR 0.69; 96.4\% CI: 0.56-0.86). Among patients with adenocarcinoma histology, the median DFS were 19.4 and 11.1 months in the nivolumab and placebo arms, respectively (HR 0.75; 95\% CI: 0.59-0.96). In squamous histology, the benefit was amplified, 29.7 versus 11.0 months with an HR of 0.61 (95\% CI: $0.42-0.88$ ). However, there are still certain issues outstanding. The subgroup analysis didn't show any advantage for gastro-oesophageal junction cancer (22.4 vs. 20.6 months; HR 0.87; 95\% CI: 0.63-1.21), ypN0 (NR vs. 27.0 months, HR 0.74; 95\% CI: 0.51-1.06), or yPT3-T4 (18.9 vs. 14.1 months, HR 0.84; 95\% CI: 0.64-1.11). More importantly, the median DFS of patients with tumors with CPS-PD-L $1<5$ did not differ between the nivolumab arm and the placebo arm (16.3 vs. 11.1 months; HR 0.89; $95 \%$ CI: $0.65-1.22)$. Conversely, the difference was statistically significant in patients with tumors with CPS $\geq 5$ (29.4 vs. 10.2 months; HR 0.62; 95\% CI: 0.46-0.83). Median OS was not yet available. In conclusion, several issues remain unanswered and there is a need to identify predictive molecular biomarkers for response to nivolumab in adjuvant setting with the aim to better select eligible patients.

\section{Expert opinion: Jaffer A. Ajani}

Based on Checkmate 577, we recommend adjuvant Nivolumab in esophageal cancer patients who have residual cancer in the resected specimen after trimodality therapy.

\section{Expert opinion: Joseph Chao}

The initial presentation at the 2020 ESMO Congress and recent publication for the CheckMate 577 trial supports the use of adjuvant nivolumab for 1 year in patients with EC specifically with lack of a complete pathologic response after neoadjuvant chemoradiotherapy and surgery (34). As this treatment approach received category 1 recommendation in NCCN guidelines, this has emerged as a standard of care at our center and received FDA approval as of May 20, 2021.

\section{Can neoadjuvant immunotherapy plus chemotherapy replace neoadjuvant radiochemotherapy in the treatment of esophageal cancer?}

\section{Expert opinion: Ken Kato}

At this time, phase I study of neo-CRT with immunotherapy for Eso Ca did not show IO's adding effect. The result of JCOG1109 will become later this year. There is the possibility of neo-chemo with triplet regimen is better than neo-CRT. If so, neo-chemo plus IO is a candidate for the new promising regimen instead of neo-CRT. JCOG1804E, which evaluates the safety and efficacy of neo-chemo combined with nivolumab, will make the answer for this.

\section{Expert opinion: Harry H. Yoon}

This remains unknown at the current time. Right now, preoperative chemotherapy or preoperative radiochemotherapy are the standard options for operable patients. Many ongoing studies are evaluating the potential benefit from adding immunotherapy to both approaches, including $\mathrm{KN}-585$.

\section{Expert opinion: Jaafar Bennouna}

There are several theorical advantages to position immunotherapy in neo-adjuvant setting, particularly in combination with chemotherapy: (I) increase the major pathological response to chemotherapy alone, (II) evaluate the response to immunotherapy on surgical specimen, allowing to better target patients for immunotherapy in adjuvant setting, and preserve patients for potential toxicities to radiotherapy. Conversely, immunotherapy in neo-adjuvant setting could induce severe auto-immune toxicities impair surgery. This multi-modal strategy should be evaluated in a randomized phase 3 trial were the standard arm would be neo-adjuvant radio-chemotherapy, surgery and nivolumab for 1 year. Stratification factors should include CPS $<5$ or $\geq 5$. 


\section{Expert opinion: Jaffer A. Ajani}

I believe this is possible as radiation's contribution is rather marginal but chemotherapy also is generally ineffective but the additional immunotherapy (especially, next generation checkpoint inhibitors) might prove very effective.

\section{Expert opinion: Joseph Chao}

This is a controversial topic and only applies to adenocarcinoma histology. Both perioperative chemotherapy and neoadjuvant chemoradiation can be considered acceptable approaches in esophageal adenocarcinoma, though our center prefers chemoradiation. Readouts from randomized trials such as ESOPEC will hopefully provide better clarity of perioperative chemotherapy versus neoadjuvant chemoradiotherapy. With readout also pending from KEYNOTE-585, there is insufficient data to indicate neoadjuvant immunotherapy plus chemotherapy can replace neoadjuvant chemoradiotherapy.

\section{After neoadjuvant radiotherapy for surgical pathology- confirmed esophageal squamous carcinoma with lymph node metastasis in the clinical setting, should the next treatment be watch-and-wait, chemotherapy, radiotherapy, or immunotherapy?}

\section{Expert opinion: Ken Kato}

The Japanese practical guideline recommended none of the adjuvant treatment for such patients after surgery. According to the result of CM-577, nivolumab has become the standard of care for such patients. But the benefit of adjuvant nivolumab is still unclear for the patients who received neoadjuvant chemotherapy before surgery.

\section{Expert opinion: Harry H. Yoon}

For tissue-confirmed ESCC with clinically staged regional nodal metastases, patients are treated with (A) definitive radiochemotherapy or (B) preoperative radiochemotherapy followed by surgery.

For patients who underwent A, the standard of care is typically to proceed with surveillance (e.g., "watch and wait"). Surveillance may include serial EGD/biopsy for patients who are believed to potentially be able to undergo future resection.

For patients who underwent $\mathrm{B}$, and who have residual tumor cells in the resected specimen, the standard in the US is adjuvant nivolumab for 1 year (see above).
For patients who underwent $\mathrm{B}$, and who have do not have residual tumor cells in the resected specimen, the standard of care is typically to proceed with surveillance.

\section{Expert opinion: Jaafar Bennouna}

For me, neo-adjuvant radiotherapy alone is not indicated.

\section{Expert opinion: Jaffer A. Ajani}

Immunotherapy. Very effective for this histology.

\section{Expert opinion: Joseph Chao}

As per item 1, if a patient does not exhibit a complete pathologic response after neoadjuvant chemoradiotherapy and surgery, regardless of squamous cell or adenocarcinoma histology, 1 year of adjuvant nivolumab immunotherapy should be the preferred approach based on the CheckMate 577 trial results.

\section{Conclusions}

Pembrolizumab has good efficacy and tolerability profiles, and has emerged as a second-line option for the treatment of PD-L1-positive locally advanced or metastatic ESCC. So far, studies have focused on its potential use as a firstline treatment, as perioperative, (neoadjuvant or adjuvant) therapy, and/or in real-world settings. Research has demonstrated that pembrolizumab has many promising applications, and further investigations into its mechanisms should be conducted.

\section{Outlook}

Controversies still exist regarding the role of pembrolizumab as an adjuvant or neoadjuvant therapy for EC, its value for patients with oncogenic mutations, and its combination with other agents (e.g., other immunotherapeutic drugs, biologics, and angiogenesis inhibitors) or radiotherapy. These issues need to be addressed in future studies.

\section{Acknowledgments}

The authors appreciate the academic support from the AME Thoracic Surgery Collaborative Group, and thank professor Ken Kato (Department of Head and Neck, Esophageal Medical Oncology, National Cancer Center Hospital, Tokyo, Japan) for the critical comments and valuable advice on this article. 
Funding: None.

\section{Footnote}

Reporting Checklist: The authors have completed the Narrative Review reporting checklist. Available at https:// dx.doi.org/10.21037/atm-21-2804

Conflicts of Interest: All authors have completed the ICMJE uniform disclosure form (available at https://dx.doi. org/10.21037/atm-21-2804). Dr. JB reports Grants for research from Astra-Zeneca, and is on the advisory board with honorarium in AstraZeneca, Bristol-Myers Squibb, F. Hoffmann-La Roche Ltd., MSD, Novartis, AMGEN, Servier, participates in the educational events with honorarium of BMS, Astra-Zeneca, Bayer, Servier, Roche, AMGEN, Daiichi, MSD. Dr. JAA serves as an Ad Hoc advisory member to Merck (paid relationship), and his institution receives research grant from Merck. Dr. JC reports grants and personal fees from Merck, personal fees from Amgen, Macrogenics, Ono Pharmaceuticals, Foundation Medicine, Daiichi-Sankyo, Bristol Myers Squibb, AstraZeneca, Astellas, Turning Point Therapeutics and Yiviva, outside the submitted work. The other authors have no conflicts of interest to declare.

Ethical Statement: The authors are accountable for all aspects of the work in ensuring that questions related to the accuracy or integrity of any part of the work are appropriately investigated and resolved.

Open Access Statement: This is an Open Access article distributed in accordance with the Creative Commons Attribution-NonCommercial-NoDerivs 4.0 International License (CC BY-NC-ND 4.0), which permits the noncommercial replication and distribution of the article with the strict proviso that no changes or edits are made and the original work is properly cited (including links to both the formal publication through the relevant DOI and the license). See: https://creativecommons.org/licenses/by-nc-nd/4.0/.

\section{References}

1. Bray F, Ferlay J, Soerjomataram I, et al. Global cancer statistics 2018: GLOBOCAN estimates of incidence and mortality worldwide for 36 cancers in 185 countries. CA Cancer J Clin 2018;68:394-424.

2. Deng HY, Zheng X, Alai G, et al. Tumor location is an independent prognostic factor of esophageal adenocarcinoma based on the eighth edition of TNM staging system in Chinese patients. Ann Transl Med 2019;7:365.

3. Lordick F, Mariette C, Haustermans K, et al. Oesophageal cancer: ESMO Clinical Practice Guidelines for diagnosis, treatment and follow-up. Ann Oncol 2016;27:v50-7.

4. Vermorken JB, Mesia R, Rivera F, et al. Platinum-based chemotherapy plus cetuximab in head and neck cancer. $\mathrm{N}$ Engl J Med 2008;359:1116-27.

5. Du F, Sun Z, Jia J, et al. Development and Validation of an Individualized Nomogram for Predicting Survival in Patients with Esophageal Carcinoma after Resection. J Cancer 2020;11:4023-9.

6. Prasad V, Kaestner V, Mailankody S. Cancer Drugs Approved Based on Biomarkers and Not Tumor TypeFDA Approval of Pembrolizumab for Mismatch RepairDeficient Solid Cancers. JAMA Oncol 2018;4:157-8.

7. Le DT, Kim TW, Van Cutsem E, et al. Phase II OpenLabel Study of Pembrolizumab in Treatment-Refractory, Microsatellite Instability-High/Mismatch Repair-Deficient Metastatic Colorectal Cancer: KEYNOTE-164. J Clin Oncol 2020;38:11-9.

8. Fiorelli A, Vitiello F, Morgillo F, et al. Pembrolizumab monotherapy in advanced NSCLC patients with low PDL1 expression: is there real evidence? Transl Cancer Res 2019;8:S618-20.

9. Li C, Zhao S, Zheng Y, et al. Preoperative pembrolizumab combined with chemoradiotherapy for oesophageal squamous cell carcinoma (PALACE-1). Eur J Cancer 2021;144:232-41.

10. Hong MH, Kim H, Park SY, et al. A phase II trial of preoperative chemoradiotherapy and pembrolizumab for locally advanced esophageal squamous cell carcinoma (ESCC). J Clin Oncol 2019;37:abstr 4027.

11. Doi T, Piha-Paul SA, Jalal SI, et al. Safety and Antitumor Activity of the Anti-Programmed Death-1 Antibody Pembrolizumab in Patients With Advanced Esophageal Carcinoma. J Clin Oncol 2018;36:61-7.

12. Shah MA, Kojima T, Hochhauser D, et al. Efficacy and Safety of Pembrolizumab for Heavily Pretreated Patients With Advanced, Metastatic Adenocarcinoma or Squamous Cell Carcinoma of the Esophagus: The Phase 2 KEYNOTE-180 Study. JAMA Oncol 2019;5:546-50.

13. Kojima T, Shah MA, Muro K, et al. Randomized Phase III KEYNOTE-181 Study of Pembrolizumab Versus Chemotherapy in Advanced Esophageal Cancer. J Clin Oncol 2020;38:4138-48. 


\section{Page 12 of 13}

14. Kato K, Sun J, Shah MA, et al. LBA8_PR -

Pembrolizumab plus chemotherapy versus chemotherapy as first-line therapy in patients with advanced esophageal cancer: The phase 3 KEYNOTE-590 study. Ann Oncol 2020;31:S1142-215.

15. Fatehi Hassanabad A, Chehade R, Breadner D, et al. Esophageal carcinoma: Towards targeted therapies. Cell Oncol (Dordr) 2020;43:195-209.

16. Carter L, Fouser LA, Jussif J, et al. PD-1:PD-L inhibitory pathway affects both CD4(+) and CD8(+) T cells and is overcome by IL-2. Eur J Immunol 2002;32:634-43.

17. Francisco LM, Sage PT, Sharpe AH. The PD-1 pathway in tolerance and autoimmunity. Immunol Rev 2010;236:219-42.

18. McDermott DF, Atkins MB. PD-1 as a potential target in cancer therapy. Cancer Med 2013;2:662-73.

19. Kwok G, Yau TC, Chiu JW, et al. Pembrolizumab (Keytruda). Hum Vaccin Immunother 2016;12:2777-89.

20. Pardoll DM. The blockade of immune checkpoints in cancer immunotherapy. Nat Rev Cancer 2012;12:252-64.

21. Freeman GJ, Long AJ, Iwai Y, et al. Engagement of the PD-1 immunoinhibitory receptor by a novel B7 family member leads to negative regulation of lymphocyte activation. J Exp Med 2000;192:1027-34.

22. Latchman Y, Wood CR, Chernova T, et al. PD-L2 is a second ligand for PD-1 and inhibits $\mathrm{T}$ cell activation. Nat Immunol 2001;2:261-8.

23. Yamamoto S, Kato K. Pembrolizumab for the treatment of esophageal cancer. Expert Opin Biol Ther 2020;20:1143-50.

24. Shapiro J, van Lanschot JJB, Hulshof MCCM, et al. Neoadjuvant chemoradiotherapy plus surgery versus surgery alone for oesophageal or junctional cancer (CROSS): long-term results of a randomised controlled trial. Lancet Oncol 2015;16:1090-8.

25. Yang H, Liu H, Chen Y, et al. Neoadjuvant Chemoradiotherapy Followed by Surgery Versus Surgery Alone for Locally Advanced Squamous Cell Carcinoma of the Esophagus (NEOCRTEC5010): A Phase III Multicenter, Randomized, Open-Label Clinical Trial. J Clin Oncol 2018;36:2796-803.

26. Joshi SS, Maron SB, Catenacci DV. Pembrolizumab for treatment of advanced gastric and gastroesophageal junction adenocarcinoma. Future Oncol 2018;14:417-30.

27. Kamath SD, Kalyan A, Benson AB 3rd. Pembrolizumab for the treatment of gastric cancer. Expert Rev Anticancer Ther 2018;18:1177-87.

28. Ninomiya K, Hotta K. Pembrolizumab for the first-line treatment of non-small cell lung cancer. Expert Opin Biol Ther 2018;18:1015-21.

29. Coupez D, Hulo P, Touchefeu Y, et al. Pembrolizumab for the treatment of colorectal cancer. Expert Opin Biol Ther 2020;20:219-26.

30. Park SY, Hong MH, Kim HR, et al. The feasibility and safety of radical esophagectomy in patients receiving neoadjuvant chemoradiotherapy with pembrolizumab for esophageal squamous cell carcinoma. J Thorac Dis 2020;12:6426-34.

31. Weinberg BA, Xiu J, Hwang JJ, et al. Immuno-Oncology Biomarkers for Gastric and Gastroesophageal Junction Adenocarcinoma: Why PD-L1 Testing May Not Be Enough. Oncologist 2018;23:1171-7.

32. Lin EM, Gong J, Klempner SJ, et al. Advances in immunooncology biomarkers for gastroesophageal cancer: Programmed death ligand 1, microsatellite instability, and beyond. World J Gastroenterol 2018;24:2686-97.

33. Catenacci DVT, Kang YK, Park H, et al. Margetuximab plus pembrolizumab in patients with previously treated, HER2-positive gastro-oesophageal adenocarcinoma (CPMGAH22-05): a single-arm, phase 1b-2 trial. Lancet Oncol 2020;21:1066-76.

34. Shah MA, Bennouna J, Doi T, et al. KEYNOTE-975 study design: a Phase III study of definitive chemoradiotherapy plus pembrolizumab in patients with esophageal carcinoma. Future Oncol 2021;17:1143-53.

35. Kato K, Cho BC, Takahashi M, et al. Nivolumab versus chemotherapy in patients with advanced oesophageal squamous cell carcinoma refractory or intolerant to previous chemotherapy (ATTRACTION-3): a multicentre, randomised, open-label, phase 3 trial. Lancet Oncol 2019;20:1506-17.

36. Kelly RJ, Ajani JA, Kuzdzal J, et al. Adjuvant Nivolumab in Resected Esophageal or Gastroesophageal Junction Cancer. N Engl J Med 2021;384:1191-203.

37. Huang J, Xu J, Chen Y, et al. Camrelizumab versus investigator's choice of chemotherapy as second-line therapy for advanced or metastatic oesophageal squamous cell carcinoma (ESCORT): a multicentre, randomised, open-label, phase 3 study. Lancet Oncol 2020;21:832-42.

38. Baxi S, Yang A, Gennarelli RL, et al. Immune-related adverse events for anti-PD-1 and anti-PD-L1 drugs: systematic review and meta-analysis. BMJ 2018;360:k793.

39. Sher AF, Golshani GM, Wu S. Fatal Adverse Events Associated with Pembrolizumab in Cancer Patients: A Meta-Analysis. Cancer Invest 2020;38:130-8.

40. Chen YL, Zhao J, Jia R, et al. Immune-related pneumonitis 
caused by programmed death-1 inhibitor Pembrolizumab: a case report and literature review. Zhonghua Jie $\mathrm{He} \mathrm{He}$ Hu Xi Za Zhi 2017;40:736-43.

41. Johnson DB, Sullivan RJ, Ott PA, et al. Ipilimumab Therapy in Patients With Advanced Melanoma and Preexisting Autoimmune Disorders. JAMA Oncol 2016;2:234-40.

42. Chuang J, Chao J, Hendifar A, et al. Checkpoint inhibition in advanced gastroesophageal cancer: clinical trial data, molecular subtyping, predictive biomarkers, and the potential of combination therapies. Transl Gastroenterol Hepatol 2019;4:63.

43. Jin Z, Yoon HH. The promise of PD-1 inhibitors in gastro-esophageal cancers: microsatellite instability vs. PD-L1. J Gastrointest Oncol 2016;7:771-88.

Cite this article as: Jin Z, Shen J, Wang C, Chen D, Zhang B, Zhang J, Ajani JA, Bennouna J, Chao J, Yoon $\mathrm{HH}$, Zhu H, Ruan Y, Zhu C, Xu A. Narrative review of pembrolizumab for the treatment of esophageal cancer: evidence and outlook. Ann Transl Med 2021;9(14):1189. doi: 10.21037/atm-21-2804
44. Zhang C, Palashati H, Tan Q, et al. Immediate and substantial evolution of $\mathrm{T}$-cell repertoire in peripheral blood and tumor microenvironment of patients with esophageal squamous cell carcinoma treated with preoperative chemotherapy. Carcinogenesis 2018;39:1389-98.

45. Lim SH, Hong M, Ahn S, et al. Changes in tumour expression of programmed death-ligand 1 after neoadjuvant concurrent chemoradiotherapy in patients with squamous oesophageal cancer. Eur J Cancer 2016;52:1-9.

46. Ajani JA, D'Amico TA, Bentrem DJ, et al. Esophageal and Esophagogastric Junction Cancers, Version 2.2019, NCCN Clinical Practice Guidelines in Oncology. J Natl Compr Canc Netw 2019;17:855-83. 